\title{
Cost Benefit Analysis of Elastic and Mixed Line Rate Optical Network Models
}

\author{
Reinhardt Rading \\ Department of Engineering and Architecture \\ University of Parma \\ Parma, Italy
}

\begin{abstract}
Network traffic continues to grow at more than 30 percent per year and we either have to install new optical infrastructures or upgrade our optical networks to meet the increasing demands. Installing new fibers seems very costly the network operators considering the heterogeneity of optical networks with some consumers requiring low bit rate and others requiring high bit rate demands. As an alternative, we can use the existing fiber infrastructure to meet the traffic demands by aggregating both high and low bit rate demands or using a single type of ratetunable technology to handle the increasing demands. This paper analyzes the two planning strategies-all period planning and incremental planning- for allocating resources in an increasing traffic and shows the pros and cons that a network operator may experience in case of using either elastic or mixed line rate optical networks.
\end{abstract}

Index Terms-Mixed Line Rate Networks, Elastic Networks,Optical Networks, Gaussian, Homogeneous.

\section{INTRODUCTION}

Network traffic continues to grow at more than 30 percent per year with Cisco predicting that by 2022, the global IP traffic will reach an all time annual rate of 4.8 zettabytes [1]. Furthermore, Cisco predicted that the average traffic per user per month will jump from 29 gigabytes in 2017 to 85 gigabytes in 2022 with a jump to over 200 gigabytes expected in the future. For example, due to Covid-19 and the consequences of the lockdown, we are witnessing a 45 percent increase in data demands even-though it is still becoming unpredictable [2]. The unpredictability of these demands means that we need a system that supports the demands reliably; optical fiber communications seems to be providing the immediate solution. The challenge arising is that we are almost reaching the capacity crunch of single mode optical fibers and therefore, the need for new technologies. Space division multiplexing (SDM) has been proposed in form of multi-core and multimode fibers, but at now researchers are working to bring the theoretical perspective to a working model.

Alternative solution is to use the existing optical network infrastructure by planning and keeping in the existing resources. Two strategies exists for planning; all period and incremental planning. All period strategy assumes that the operator knows the quantity of increasing demands from period $T_{0}$ to period $T_{4}$. Using the knowledge of the traffic evolution, the operator decides where to install additional network resources at the start of the period $T_{0}$. In incremental planning, the operator analyzes the increase in demands at each period and deploy resources in cases only where an increase in demands was observed.

A light path can travel several kilometres of distance depending on the physical impairments [3] along the light path. The higher the bit rate, the lesser the maximum distance [4] the signal can travel based on a threshold bit error rate (BER) [5]. Combining high capacity short reach light paths with low capacity short reach has been proven as an efficient way to handle the various lengths scales and the range of capacity demands.

Mixed line rate (MLR) and elastic (EON) optical networks are cost efficient models that can be used with the already existing optical infrastructure to meet the increasing demands. MLR relies on different technologies for each datarate, for example, using on-off keying modulation format for $10 \mathrm{~Gb} / \mathrm{s}, 40$ $\mathrm{Gb} / \mathrm{s}$ differential phase shift keying, and polarization division multiplexing-quadrature modulation for $100 \mathrm{~Gb} / \mathrm{s}$. EON uses a a single re-configurable rate technology to handle all the demands and this simplifies the design of the optical network by allowing optimal sharing of resources though at the expense of high price per piece of the devices.

This paper analyzes the cost advantage of using either MLR or EON networks dependent on the choice of the network upgrade(all period or incremental) scenario. The cost is considered in terms of opto-electronic interfaces where the total cost of the network is provided by the number of total devices deployed at each time period, multiplied by their cost,and scaled by the cost erosion. I have assumed that all transported rates occupy the same spectrum slots and thus the resources considered are only the $3 \mathrm{R}$ devices. It is divided into three sections, Section II discusses the network model, section III discusses the simulation results, and finally, section IV discusses the conclusion.

\section{NETWORK MODEL}

The National DT with 14 nodes and 23 links and Continental US with 50 nodes and 65 links network scenarios are considered for this study. Each network scenario is analyzed using the DIAMOND tool where an assumption that each fiber is a single mode fiber carrying 96 wavelengths was made.

The tool calculates and determines the shortest path, the suitable rates,and determines which $3 \mathrm{R}$ optoelectronic devices 
are needed in cases where wavelength constraints is experienced and or regeneration is required. If a connection is deemed unsuitable, it is blocked. The tool selects the most cost efficient path. For example, there exists several solutions to route a $90 \mathrm{~Gb} / \mathrm{s}$ demand dependent on the distance, number of rates required to perform the routing, among others. A 90 $\mathrm{Gb} / \mathrm{s}$ may be routed as $1 \times 100 \mathrm{~Gb} / \mathrm{s}, 3 \times 40 \mathrm{~Gb} / \mathrm{s}, 9 \times 10 \mathrm{~Gb} / \mathrm{s}$ or as $2 \times 40 \mathrm{~Gb} / \mathrm{s}+10 \mathrm{~Gb} / \mathrm{s}$.

The initial traffic load at $T_{0}$ is assumed to be $10 \mathrm{~Tb} / \mathrm{s}$ evolving from $T_{0}$ to $T_{4}\left(T_{0}, T_{1}, T_{2}, T_{3}, T_{4}\right)$ with an annual increase of each demand by a random amount uniformly drawn in the range between 10 to 30 percent. We analyze the two strategies using both homogeneous and Gaussian traffic matrix distribution. In homogeneous distribution, all the nodes of demand have the same probability with a granularity of 5 $\mathrm{Gb} / \mathrm{s}$. At each pair of node, if there exists a demand at $T_{i-1}$, the demand at time $T_{i}$ is 1.2 percent of the demand at $T_{i-1}$. If no demand existed at time $T_{i-1}$ but its requested at time $T_{i}$, the demand is set at $5 \mathrm{~Gb} / \mathrm{s}$.

For Gaussian distribution, the matrix is made of randomly drawn node pairs with each drawn pairs having a uniform distribution with a mean of $30 \mathrm{~Gb} / \mathrm{s}$ and a standard deviation of $5 \mathrm{~Gb} / \mathrm{s}$. The same demand changes are experienced as explained in homogenous case from time $T_{i-1}$ to $T_{i}$.

We assume that the reach of 10, 40 and 100 rates of MLR are $5000 \mathrm{~km}, 2000 \mathrm{~km}$, and $800 \mathrm{~km}$ respectively and the cost of opto-electronic interfaces are in the ratio of 1:3:7 at $T_{0}$. Due to the rapid technological development, we assume that the cost erosion and the price of the $3 \mathrm{R}$ devices changes from 1:3:7 at $T_{0}$ to $1: 1.8: 2.5$ at $T_{4}$. At time $T_{1}$ the cost erosion is in the ratio $1: 2.8: 4.8$, at time $T_{2}$ is $1: 2.5: 3.8$ whereas at time $T_{3}$ the ratio is $1: 2: 2.8$.

The cost-erosion is not constant for all the devices, but depend on the technology maturity. The technology of $10 \mathrm{~Gb} / \mathrm{s}$ is currently in its maturity and hence its cost erosion will be negligible with respect to the cost erosion of other rates and elastic devices. The cost for an MLR network model is given by the number of opto-electronic devices deployed at each time period, multiplied by the cost erosion.

TABLE I

THE COST OF OPTO-ELECTRONIC INTERFACE IN MLR NETWORK MODEL

\begin{tabular}{|c|c|c|}
\hline Data rates in Gb/s & Cost(units) & Reach $(\mathrm{km})$ \\
\hline 10 & 1 & 5000 \\
\hline 40 & 3 & 2000 \\
\hline 100 & 7 & 800 \\
\hline
\end{tabular}

Eventhough adaptable transponders have the ability to accomodate higher rates, we assume that the rates at $10 \mathrm{~Gb} / \mathrm{s}$ and 40 $\mathrm{Gb} / \mathrm{s}$ has the same functionality as MLR rates. Their reaches are $5000 \mathrm{~km}, 2000 \mathrm{~km}$ and $1200 \mathrm{~km}$ for the $100 \mathrm{~Gb} / \mathrm{s}$ rate. It can be noted that in EON case, the $100 \mathrm{~Gb} / \mathrm{s}$ rate has a higher reach compared to the MLR case because of the absence of $10 \mathrm{~Gb} / \mathrm{s}$ on-off keying rate which is detrimental to the reach of the $100 \mathrm{~Gb} / \mathrm{s}$ rate thus no mixing between MLR and EON devices. We assume that EON is yet to reach its maturity and therefore experiences a cost degradation from 4.8 at $T_{0}$ to 2.5 at $T_{4}$. The cost of the EON network model is given by the number of deployed devices multiplied by the cost erosion and only taking into account the resources and devices deployed at the specific time evolution.

TABLE II

THE COST OF OPTO-ELECTRONIC INTERFACES IN EON NETWORK MODEL

\begin{tabular}{|c|c|c|}
\hline Data rates in Gb/s & Cost(units) & Reach $(\mathrm{km})$ \\
\hline 10 & 7 & 5000 \\
\hline 40 & 7 & 2000 \\
\hline 100 & 7 & 1200 \\
\hline
\end{tabular}

In the MLR model, the planning tool checks the demand for a particular node pair at time $T_{i}$ (where $i>0$ ) if it existed a spare capacity from time $T_{i-1}$. The existing spare capacity is used to route the requested capacity at time $T_{i-1}$ and if there exists further needed capacity, resources are allocated to the new demand with the same cost minimization method simulated during time $T_{0}$ but accounting for the price degradation.

In the EON case, there exists the advantage of using the flexibility of the transponder. At each subsequent evolution time $T_{i}$, the cost minimization method at time $T_{0}$ is simulated, but accounting for resources already deployed at earlier time intervals and the price degradation of the new resources.

\section{Simulation Results}

Twenty seeds were drawn for each simulation and their results averaged and thus these observation are from the averaged results.

Starting with the first strategy-incremental planning-we can notice that EON model experiences a higher capital expenditure (35 percent higher) cost requirement compared to the MLR model at time $T_{0}$ as shown on figure 1 . In the subsequent time intervals, a cost reduction in EON model is observed whereas an increase in expenditure cost is observed in the MLR model.

This observation can be explained as resulting from the availability of low data rate transponders which cost less compared to the EON network model due to technology maturity . It can be stipulated that several connections at $T_{0}$ were requested at the rate of $10 \mathrm{~Gb} / \mathrm{s}$ for both model. The 10 $\mathrm{Gb} / \mathrm{s}$ can be routed cheaply at MLR compared to EON where several $10 \mathrm{~Gb} / \mathrm{s}$ connections are routed but the at cost of 100 $\mathrm{Gb} / \mathrm{s}$ and thus the cost of $100 \mathrm{~Gb} / \mathrm{s}$ transponders must be met at $T_{0}$.

As the time evolves, EON networks become more cost efficient compared to MLR network from time $T_{1}$ to $T_{4}$. This can be explained that the installed adaptive transponders in EON model can be tuned to accept more datarate as the demands increases. The high cost in MLR model can be explained as resulting from the necessity to route different datarates as the demand grows. The high data rates light path in MLR model have short reaches hence requires regeneration 


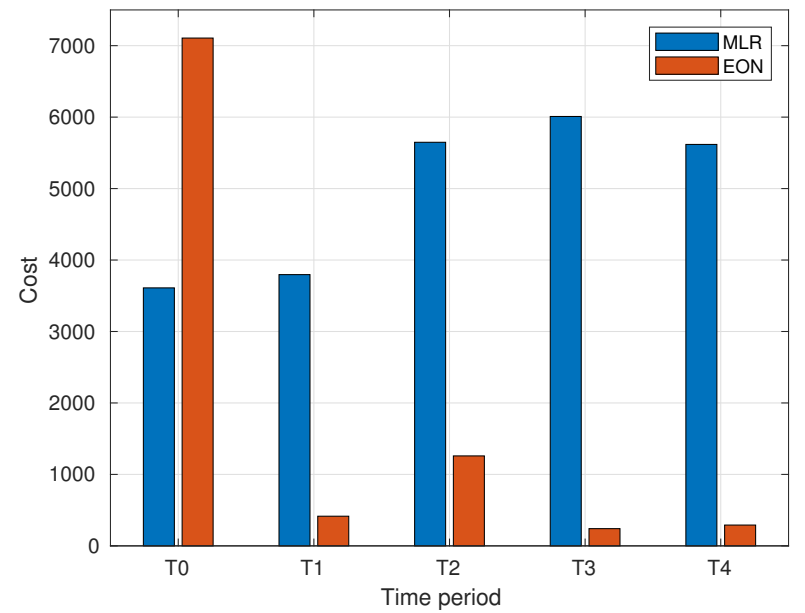

Fig. 1. The cost analysis of opto-electronic interfaces in a Gaussian traffic matrix of Continental US network scenario pitting EON vs MLR from period $T_{0}$ to $T_{4}$. Incremental planning strategy is analyzed.

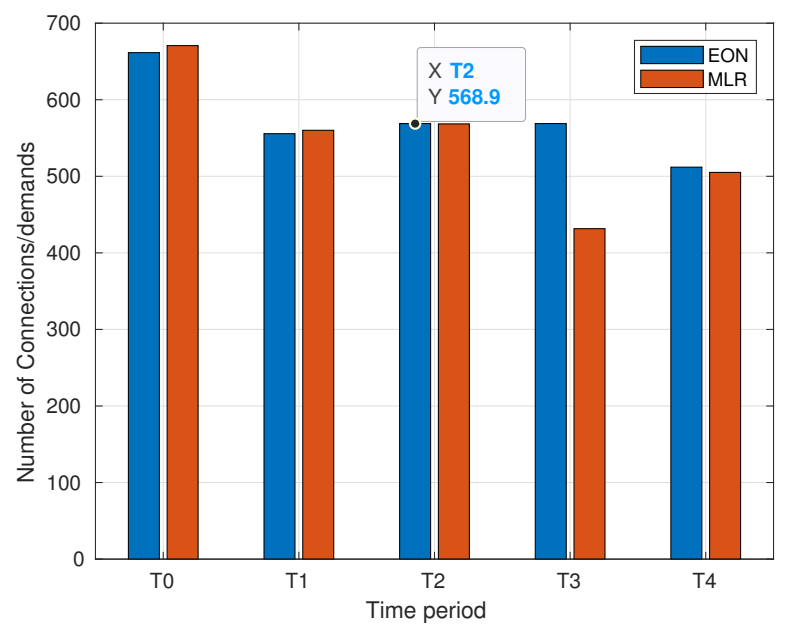

Fig. 2. The number of connections of a Gaussian traffic matrix of Continental US network scenario pitting EON vs MLR from period $T_{0}$ to $T_{4}$

therefore adding additional cost to the model arising from $3 \mathrm{R}$ devices. The different datarates also leads to spectrum waste as there is the need to route the different rates in separate bands (This assumption is ignored in this simulation).

The number of connections routed is high at $T_{0}$ and from time $T_{1}$, it reduces both for MLR and EON from as shown in figure 2 . This validates that several low rates were demanded at $T_{0}$ hence better cost at MLR and high costs at EON. As time evolves, additional connections and resources are required to meet the demands at MLR whereas after tuning, the elastic transponders readily meets the increasing demands.

The number of blocked demand is highly experienced at mixed line rate model. For example, when simulating the $\mathrm{Na}$ tional DT using both Gaussian and homogeneous traffic matrices, we experienced zero blockings when in EON scenario. An

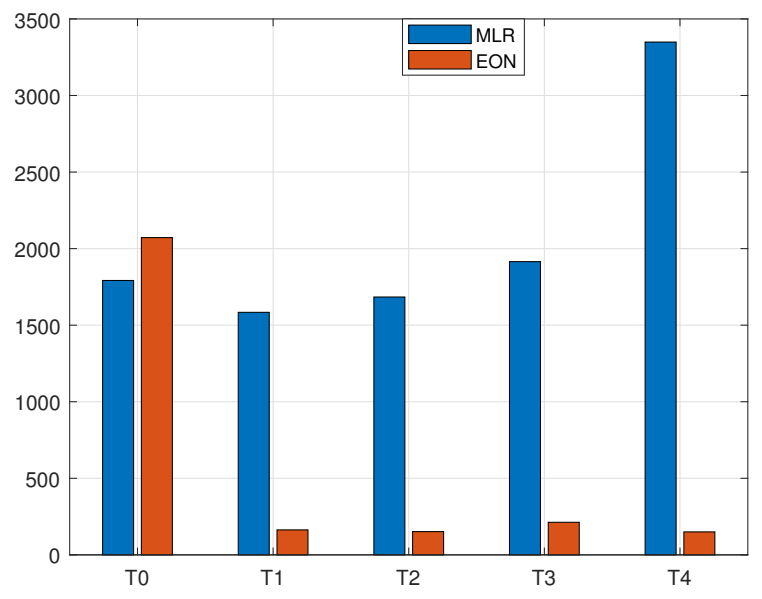

Fig. 3. The cost analysis of opto-electronic interfaces in a Gaussian traffic matrix of National DT network scenario pitting EON vs MLR from period $T_{0}$ to $T_{4}$. Incremental planning is analyzed.

average of 26 connections are blocked when in MLR scenario under the same conditions for the entire time evolution. As the demands increases, connections with different rates and reaches are routed. The connections with shorter reaches will need regeneration and this creates complexities as the number of connections increases leading to some connections being dropped.

When the simulation was repeated using incremental strategy for National DT network of Gaussian traffic matrix, we made similar observations as in the Continental US network. EON model has a higher initial cost at $T_{0}$ but reduces in the subsequent times whereas the opposite is observed for MLR network as shown in figure 3 .

When we considered the homogenous traffic matrix of Continental US, its result is as shown in figure 4. EON network model is expenditure extensive at time $T_{0}$ but this reduces as the time evolves. At time $T_{3}$, we observed a negative cost which is in this case assumed to be zero since the negative cost arises from taking into account the already existing resources. Unlike MLR, the cost is relatively cheaper but increases as time evolves. At the end of the cycle at $T_{4}$, EON model is 32 percent cheaper than MLR model.

Figure 6 depicts the total cost of opto-electronic devices for National DT network realized from time $T_{0}$ to $T_{4}$, figure 8 depicts total cost of opto-electronic devices incurred for routing Continental AN scenario under incremental planning strategy. We can conclude from the above observations that EON network models is cheaper compared to MLR network models. EON has a higher initial expenditure in both Gaussian and homogeneous traffic but reduces significantly as both the demands and the time evolves. On the other hand we can notice that MLR model has a lower initial cost but as time and the demand increases, the cost increases. This is because as the demand increases, different rates of high values are required and thus additional devices. The $100 \mathrm{~Gb} / \mathrm{s}$ in MLR has 


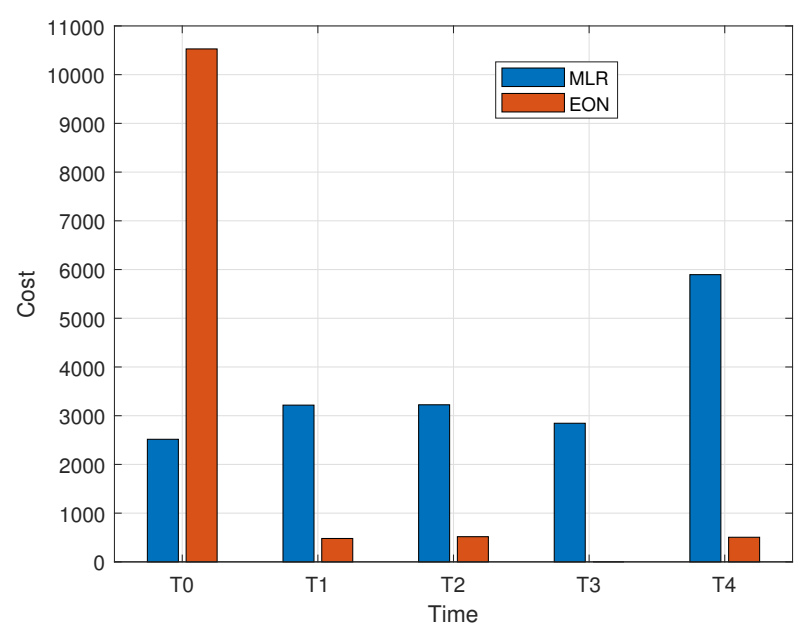

Fig. 4. The cost analysis of opto-electronic interfaces in a Homogeneous traffic matrix of Continental US network scenario pitting EON vs MLR from period $T_{0}$ to $T_{4}$. Increasing planning strategy is analyzed

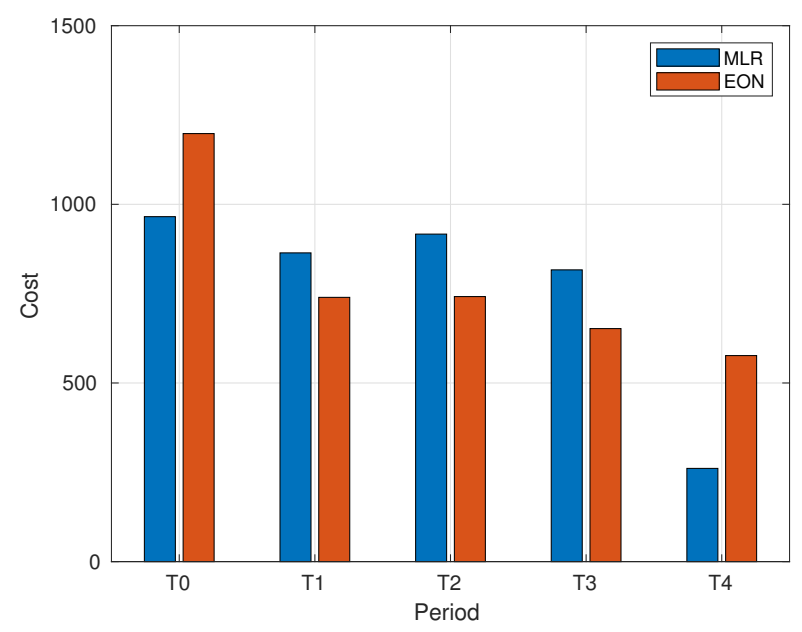

Fig. 5. The cost analysis of opto-electronic interfaces in a Homogeneous traffic matrix of National DT network scenario pitting EON vs MLR from period $T_{0}$ to $T_{4}$. All period planning strategy is analyzed

a shorter reach and therefore regeneration is needed in cases where the length is longer than the reach and this increases the network cost.

EON meanwhile, we have to meet the initial higher cost of installing $100 \mathrm{~Gb} / \mathrm{s}$ even when the demands are lower. As demands increases, the transponders can be reconfigured to to accommodate the demands with no additional devices.

Secondly, we analyzed all period strategy. Simulating National DT network made up of homogenous traffic matrix, its result is shown on figure 5 .

A higher EON network cost is observed at time $T_{0}$ for an all period strategy; similar to what was observed in incremental strategy. As the time evolves, the EON cost was observed to be lower than that of MLR.

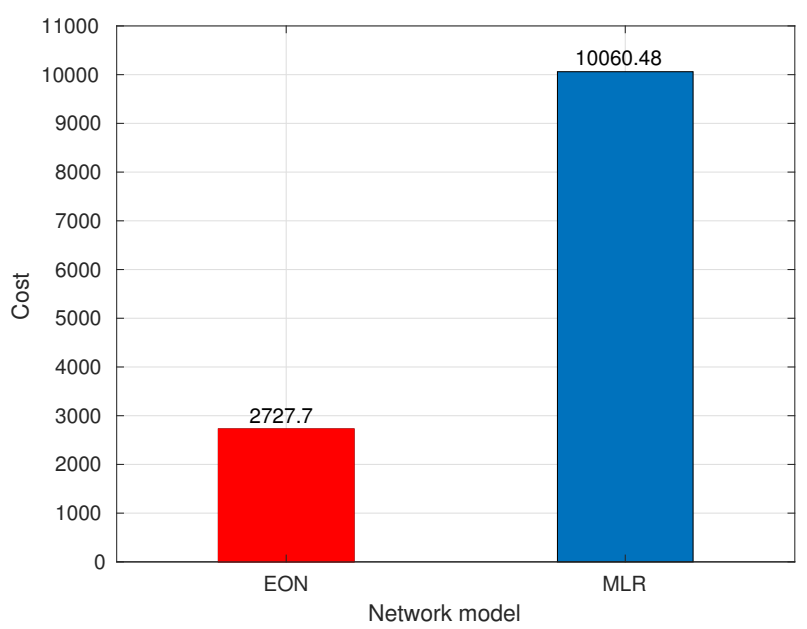

Fig. 6. The total cost of EON vs MLR Network model for National DT network scenario realized using incremental planning strategy, Gaussian traffic matrix

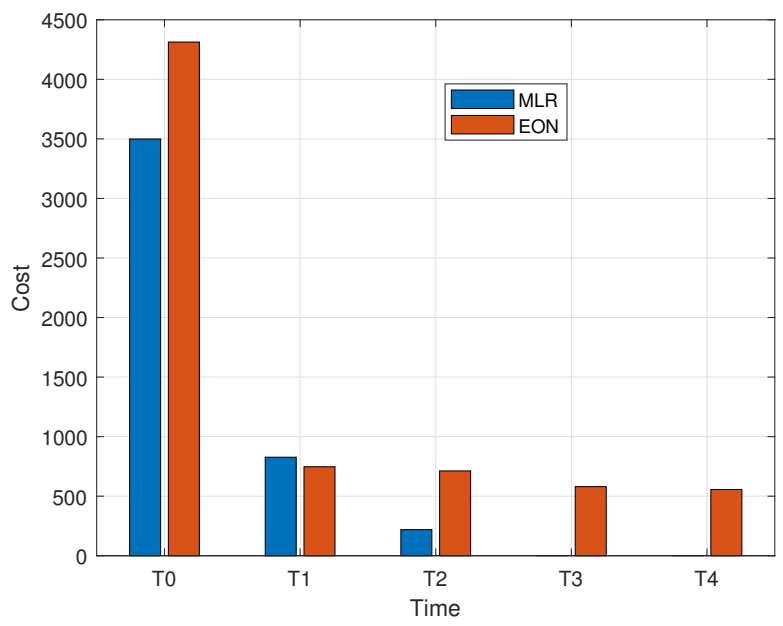

Fig. 7. The total cost of opto-electronic interfaces in EON vs MLR Network model for a Gaussian traffic matrix in the Continental US network realized using all period planning strategy

Figure 7 shows the results got when Continental US network is simulated for all period planning using Gaussian matrix. We can observe a higher cost at time $T_{0}$ for EON model compared to MLR model and a reduction in cost is observed in the subsequent timelines. In MLR model, we observed zero connections resulting from blockages at $T_{3}$ and $T_{4}$. Comparing the cost of devices realized as a result of incremental strategy as shown in figure 6 versus all period strategy as shown in figure 9 , we observe a cheaper option in all period strategy than in incremental strategy. This seems advantageous and might attract the network operator but as time evolves, the planning made at time $T_{0}$ turns out to be constraint in increasing the network size as observed in MLR model at time $T_{3}$ and $T_{4}$ as shown in figure 7 where several blockages were recorded.

Incremental planning allows the possibility of assessing the 


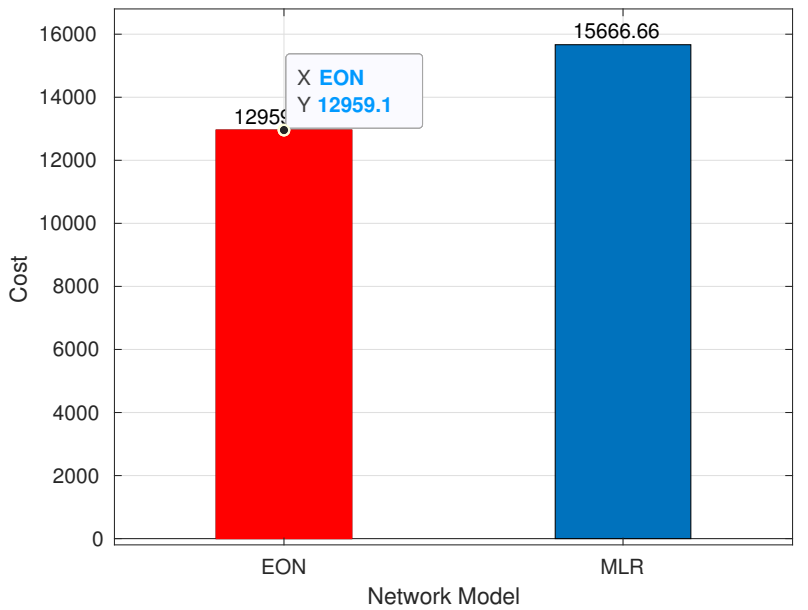

Fig. 8. The total cost of opto-electronic interfaces in EON vs MLR Network model for Continental US realized using incremental planning strategy

status of the network at each time interval and decide which resources to deploy. This favours both MLR and EON model as depicted in this report.

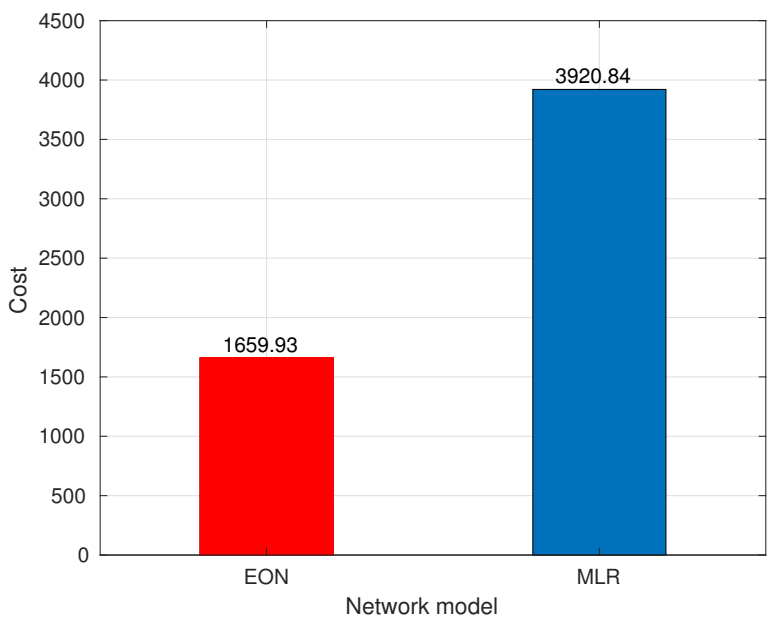

Fig. 9. The total cost of opto-electronic interfaces in EON vs MLR Network model of National DT realized using all period planning strategy, Gaussian traffic matrix

\section{CONCLUSION}

This paper has shown that elastic network model is 32 percent more cost efficient compared to the mixed line network model. The adaptable transponders allows the flexibility in cases where demands are increasing with time evolution. Unlike mixed line rate, elastic networks have a higher capital expenditure but reduces with time evolution whereas for mixed line rate, the initial capital expenditure is relatively low but is relatively higher in the successive periods.

All period planning strategy seems advantageous in terms of cost but it introduces network constraint as time evolves.
Incremental planning is an efficient option for both MLR and EON model.

\section{REFERENCES}

[1] Cisco Visual Networking Index: Forecast and Trends, 2017-2022, shorturl.at/gijrB

[2] Network traffic insights in the time of COVID-19: April 9 update, https://www.nokia.com/blog/network-traffic-insights-time-covid19-april-9-update

[3] R.Rading, "Evaluation of Nonlinear Interference Effects in a Dispersion Managed (DM) Optical Fiber: Performance and Transmission Analysis of 16QAM Modulation using Split Step Fourier Method", https://doi.org/10.36227/techrxiv.13102442, 2020

[4] F. Rambach, et al. ,"A multilayer cost model for metro/core networks," IEEEIOSA Journal of Optical Communications and Networking, vol. 5, no. 3 , pp. 210-225, 2013.

[5] J.M Simmons, Optical Network Design and Planning, Springer 2008. 